PROCEEDINGS OF THE

AMERICAN MATHEMATICAL SOCIETY

Volume 130, Number 8, Pages 2351-2361

S 0002-9939(02)06355-4

Article electronically published on March 12, 2002

\title{
SHARP LOCAL ISOPERIMETRIC INEQUALITIES INVOLVING THE SCALAR CURVATURE
}

\author{
OLIVIER DRUET
}

(Communicated by Jozef Dodziuk)

\begin{abstract}
We provide sharp local isoperimetric inequalities on Riemannian manifolds involving the scalar curvature, and thus answer a question asked by Johnson and Morgan.
\end{abstract}

\section{IntRoduCtion AND STATEMENT OF THE RESUlts}

Let $(M, g)$ be a complete Riemannian manifold of dimension $n \geq 2$ with sectional curvature $K_{g} \leq K_{0}$. A long-standing conjecture, a formulation of which can be found in [1], asserts that for any $x \in M$, there exists $r_{x}>0$ such that for any $\Omega$ contained in the geodesic ball of center $x$ and radius $r_{x}$,

$$
|\partial \Omega|_{g} \geq|\partial B|_{g_{0}}
$$

where $||$.$g (resp. |\cdot|_{g_{0}}$ ) denotes the volume with respect to $g$ (resp. $\left.g_{0}\right)$ and $B$ is a ball of volume $|\Omega|_{g}$ in the model space $\left(M_{0}, g_{0}\right)$ of constant sectional curvature $K_{0}$. A compact version of this conjecture was proved, with an additional assumption on the Gauss-Bonnet-Chern integrand in even dimensions, in the very nice Johnson and Morgan [10]. A natural question that Johnson and Morgan [10] asked is the following: is the result still true if we assume that the scalar curvature of $(M, g)$ is such that $S_{g}<n(n-1) K_{0}$ instead of assuming that $K_{g} \leq K_{0}$ ? We answer this question in the affirmative and prove the following:

Theorem 1. Let $(M, g)$ be a complete Riemannian manifold of dimension $n \geq 2$ and let $x \in M$. Assume that $S_{g}(x)<n(n-1) K_{0}$ for some $K_{0} \in \mathbf{R}$. Then there exists $r_{x}>0$ such that for any $\Omega$ contained in the geodesic ball of center $x$ and radius $r_{x}$,

$$
|\partial \Omega|_{g}>|\partial B|_{g_{0}}
$$

where $B$ is a ball of volume $|\Omega|_{g}$ in the model space $\left(M_{0}, g_{0}\right)$ of constant sectional curvature $K_{0}$.

In the compact setting, the situation that was actually considered by Johnson and Morgan [10], we have the following:

Received by the editors March 15, 2001.

2000 Mathematics Subject Classification. Primary 49J40, 53C21.

(C)2002 American Mathematical Society 
Theorem 2. Let $(M, g)$ be a compact Riemannian manifold of dimension $n \geq 2$ with scalar curvature $S_{g}<n(n-1) K_{0}$. There exists $V>0$ such that for any subset $\Omega$ of $M$ of volume less than or equal to $V$,

$$
|\partial \Omega|_{g}>|\partial B|_{g_{0}}
$$

where $B$ is a ball of volume $|\Omega|_{g}$ in the model space $\left(M_{0}, g_{0}\right)$ of constant sectional curvature $K_{0}$.

These results are optimal in the following sense: if we only assume that the Ricci curvature of $M$ verifies $R i c_{g} \leq(n-1) K_{0}$, the above isoperimetric comparison fails. Indeed, for any $n$-manifold $M$ which is Ricci-flat but not flat (see 3] for examples of such manifolds), one may find a ball $B_{r}$ in $M$ of radius $r$ as small as we want which verifies

$$
\left|\partial B_{r}\right|_{g}<|\partial B|_{\xi}
$$

where $B$ is a ball of volume $\left|B_{r}\right|_{g}$ in the Euclidean space $\left(\mathbf{R}^{n}, \xi\right)$. The above comparison result is also false on $S^{2} \times S^{2}$, as noticed in [10. The proof of Theorem 1 is based on the study of local optimal Sobolev inequalities. The proof relies on PDE techniques and a fine asymptotic analysis of solutions of quasi-elliptic equations involving the $p$-Laplacian. Theorem 2 is a consequence of Theorem 1 thanks to geometric measure theory. The relevance of the scalar curvature when studying the validity of sharp Sobolev inequalities was noticed first by the author in [4] and underlined by Hebey in [9].

\section{Sobolev inequalities And Proof of Theorem 1}

Let $B$ be a ball in the model space $\left(M_{0}, g_{0}\right)$ of constant sectional curvature $K_{0}$. It is not difficult to check that, for balls of small volume,

$$
|\partial B|_{g_{0}}^{2}=K(n, 1)^{-2}|B|_{g_{0}}^{2 \frac{n-1}{n}}-\frac{n}{n+2}\left(n(n-1) K_{0}\right)|B|_{g_{0}}^{2}+o\left(|B|_{g_{0}}^{2}\right) .
$$

Here, $n=\operatorname{dim} M_{0}$ and

$$
K(n, 1)^{-1}=n\left(\frac{\omega_{n-1}}{n}\right)^{\frac{1}{n}} .
$$

Now, let $(M, g)$ be a complete Riemannian manifold of dimension $n \geq 2$ and let $x_{0} \in M$. In order to prove Theorem 1 , it is clearly sufficient to prove that for any $\varepsilon>0$, there exists $r_{\varepsilon}>0$ such that for any $\Omega \subset B_{g}\left(x_{0}, r_{\varepsilon}\right)$,

$$
|\partial \Omega|_{g}^{2} \geq K(n, 1)^{-2}|\Omega|_{g}^{2 \frac{n-1}{n}}-\left(\frac{n}{n+2} S_{g}\left(x_{0}\right)+\varepsilon\right)|\Omega|_{g}^{2} .
$$

It is now well known that (2.1) is a consequence of the following Sobolev inequality: for any $u \in C_{c}^{\infty}\left(B_{g}\left(x_{0}, r_{\varepsilon}\right)\right)$,

$$
\|u\|_{\frac{n}{n-1}}^{2} \leq K(n, 1)^{2}\left(\|\nabla u\|_{1}^{2}\right)+\left(\frac{n}{n+2} S_{g}\left(x_{0}\right)+\varepsilon\right)\|u\|_{1}^{2}
$$

where $\|\cdot\|_{p}$ denotes the $L^{p}$-norm with respect to the Riemannian volume element $d v_{g}$. Indeed, $\Omega \subset B_{g}\left(x_{0}, r_{\varepsilon}\right)$ being given, one may find a sequence $\left(u_{i}\right)$ of smooth functions with compact support in $B_{g}\left(x_{0}, r_{\varepsilon}\right)$ such that for any $q \geq 1$,

$$
\lim _{i \rightarrow+\infty} \int_{B_{g}\left(x_{0}, r_{\varepsilon}\right)}\left|u_{i}\right|^{q} d v_{g}=|\Omega|_{g}
$$


and

$$
\lim _{i \rightarrow+\infty} \int_{B_{g}\left(x_{0}, r_{\varepsilon}\right)}\left|\nabla u_{i}\right|_{g} d v_{g}=|\partial \Omega|_{g}
$$

Before starting the proof of the above Sobolev inequality, we must set up some notations. For any $1 \leq p<n$, we let

$$
K(n, p)^{-p}=\inf _{u \in C_{c}^{\infty}\left(\mathbf{R}^{n}\right), u \neq 0} \frac{\int_{\mathbf{R}^{n}}|\nabla u|_{\xi}^{p} d v_{\xi}}{\left(\int_{\mathbf{R}^{n}}|u|^{p^{\star}} d v_{\xi}\right)^{\frac{p}{p^{\star}}}}
$$

where $p^{\star}=\frac{n p}{n-p}$ is the critical exponent for the Sobolev embeddings and $\xi$ is the Euclidean metric. The value of $K(n, p)$ is explicitly known (see [1] or [15]) but the only point of interest to us is that

$$
\lim _{p \rightarrow 1} K(n, p)=K(n, 1)=\frac{1}{n}\left(\frac{n}{\omega_{n-1}}\right)^{\frac{1}{n}} .
$$

We also let, for $1 \leq p<n, H_{1}^{p}\left(\mathbf{R}^{n}\right)$ be the standard Sobolev space of order $p$, that is the completion of $C_{c}^{\infty}\left(\mathbf{R}^{n}\right)$ for the norm

$$
\|u\|_{H_{1}^{p}}=\left(\int_{\mathbf{R}^{n}}|\nabla u|_{\xi}^{p} d v_{\xi}\right)^{\frac{1}{p}} .
$$

At last, we let $B V\left(\mathbf{R}^{n}\right)$ be the space of functions with bounded variations in $\mathbf{R}^{n}$, defined as the completion of $C_{c}^{\infty}\left(\mathbf{R}^{n}\right)$ with respect to the norm

$$
\|u\|_{B V}=\sup \left\{-\int_{\mathbf{R}^{n}} u \operatorname{div}(X) d v_{\xi},\|X\|_{L^{\infty}\left(\mathbf{R}^{n}\right)} \leq 1, \operatorname{div}(X) \in L^{n}\left(\mathbf{R}^{n}\right)\right\}
$$

where $\operatorname{div}(X)=\partial^{i} X_{i}$. Basic facts about $B V\left(\mathbf{R}^{n}\right)$ can be found in [7] or [16].

As already mentioned, Theorem 1 reduces to the following proposition:

Proposition. Let $(M, g)$ be a complete Riemannian manifold of dimension $n \geq 2$ and let $x_{0} \in M$. For any $\varepsilon>0$, there exists $r_{\varepsilon}>0$ such that for any $u$ in $C_{c}^{\infty}\left(B_{g}\left(x_{0}, r_{\varepsilon}\right)\right)$,

$$
\|u\|_{\frac{n}{n-1}}^{2} \leq K(n, 1)^{2}\left(\|\nabla u\|_{1}^{2}+\alpha_{\varepsilon}\|u\|_{1}^{2}\right)
$$

where $\alpha_{\varepsilon}=\frac{n}{n+2} S_{g}\left(x_{0}\right)+\varepsilon$.

We prove the Proposition in what follows.

Proof of the Proposition. Clearly, we may assume, without loss of generality, that $M=\mathbf{R}^{n}$ and that $x_{0}=0$. We let, for any $r>0$, any $p>1$ and any $\varepsilon>0$,

$$
\lambda_{p, r}=\inf _{u \in C_{c}^{\infty}\left(B_{g}(0, r)\right), u \neq 0} \frac{\left(\int_{B_{g}(0, r)}|\nabla u|_{g}^{p} d v_{g}\right)^{\frac{2}{p}}+\alpha_{\varepsilon}\left(\int_{B_{g}(0, r)}|u|^{p} d v_{g}\right)^{\frac{2}{p}}}{\left(\int_{B_{g}(0, r)}|u|^{p^{\star}} d v_{g}\right)^{\frac{2}{p^{\star}}}} .
$$

We proceed by contradiction. We assume that there exists $\varepsilon_{0}>0$ such that for any $r>0$,

$$
\lambda_{1, r}<K(n, 1)^{-2} .
$$


Then, since $\lim \sup _{p \rightarrow 1} \lambda_{p, r} \leq \lambda_{1, r}$, one easily gets that for any $r>0$, there exists $p_{r}>1$ such that

$$
\lambda_{p_{r}, r}<K(n, 1)^{-2}\left(\frac{n-p_{r}}{p_{r}(n-1)}\right)^{2}, \lambda_{p_{r}, r}<K\left(n, p_{r}\right)^{-2} .
$$

We may assume that $r \rightarrow 0$ and we may choose $p_{r}$ decreasing when $r$ is decreasing. Then we get a sequence $p>1$ going to 1 and a sequence $r_{p}>0$ going to 0 as $p$ goes to 1 which verify (2.2). It is by now classical that the second inequality in (2.2) ensures the existence of a minimizer $u_{p}$ which satisfies the following:

$$
\begin{aligned}
& C_{p} \Delta_{p} u_{p}+\alpha\left\|u_{p}\right\|_{p}^{2-p} u_{p}^{p-1}=\lambda_{p} u_{p}^{p^{\star}-1} \text { in } B_{g}\left(0, r_{p}\right), \\
& u_{p} \in C^{1, \eta}\left(B_{g}\left(0, r_{p}\right)\right) \text { for some } \eta>0, \\
& u_{p}>0 \text { in } B_{g}\left(0, r_{p}\right), u_{p}=0 \quad \text { on } \partial B_{g}\left(0, r_{p}\right), \\
& \int_{B_{g}\left(0, r_{p}\right)} u_{p}^{p^{\star}} d v_{g}=1, \\
& \lambda_{p}<K(n, p)^{-2}, \lambda_{p}<K(n, 1)^{-2}\left(\frac{n-p}{p(n-1)}\right)^{2}, \\
& C_{p}=\left(\int_{B_{g}\left(0, r_{p}\right)}\left|\nabla u_{p}\right|_{g}^{p} d v_{g}\right)^{\frac{2-p}{p}} .
\end{aligned}
$$

In the above equations, $\Delta_{p}$ is the $p$-laplacian with respect to $g$, that is $\Delta_{p} u=$ $-\operatorname{div}_{g}\left(|\nabla u|_{g}^{p-2} \nabla u\right)$, and we have set

$$
\alpha=\frac{n}{n+2} S_{g}(0)+\varepsilon_{0} .
$$

Now the aim is to study this sequence $\left(u_{p}\right)$ as $p \rightarrow 1$. We let $x_{p}$ be a point in $B_{g}\left(0, r_{p}\right)$ where $u_{p}$ achieves its maximum and we also let

$$
u_{p}\left(x_{p}\right)=\mu_{p}^{1-\frac{n}{p}} .
$$

We have

$$
1=\int_{B_{g}\left(0, r_{p}\right)} u_{p}^{p^{\star}} d v_{g} \leq \operatorname{Vol}_{g}\left(B_{g}\left(0, r_{p}\right)\right) \mu_{p}^{-n}
$$

and since $r_{p}$ goes to $0, \mu_{p}$ goes to 0 as $p$ goes to 1 . In the same way, thanks to Hölder's inequalities, we get

$$
\lim _{p \rightarrow 1} \int_{B_{g}\left(0, r_{p}\right)} u_{p}^{p} d v_{g}=0 .
$$

Step 1. We first claim that

$$
\lim _{p \rightarrow 1} \lambda_{p}=K(n, 1)^{-2}
$$

and that

$$
\lim _{p \rightarrow 1} \int_{B_{g}\left(0, r_{p}\right)}\left|\nabla u_{p}\right|_{g}^{p} d v_{g}=K(n, 1)^{-1} .
$$


Indeed (see for instance 8] for an exposition in book form) for any $\varepsilon>0$ there exists $B_{\varepsilon}>0$ such that for any $p>1$,

$$
\begin{aligned}
\left(\int_{B_{g}\left(0, r_{p}\right)} u_{p}^{p^{\star}} d v_{g}\right)^{2 \frac{n-1}{n}} \leq & (K(n, 1)+\varepsilon)^{2}\left(\int_{B_{g}\left(0, r_{p}\right)}\left|\nabla\left(u_{p}^{\frac{p(n-1)}{n-p}}\right)\right|_{g} d v_{g}\right)^{2} \\
& +B_{\varepsilon}\left(\int_{B_{g}\left(0, r_{p}\right)} u_{p}^{\frac{p(n-1)}{n-p}} d v_{g}\right)^{2}
\end{aligned}
$$

which gives with (2.3), (2.4) and Hölder's inequalities

$$
1 \leq(K(n, 1)+\varepsilon)^{2}\left(\frac{p(n-1)}{n-p}\right)^{2}\left(\lambda_{p}-\alpha\left\|u_{p}\right\|_{p}^{2}\right)+B_{\varepsilon}\left\|u_{p}\right\|_{p}^{2}
$$

This leads with (2.7) to

$$
1 \leq\left(1+\varepsilon K(n, 1)^{-1}\right)^{2} \liminf _{p \rightarrow 1}\left(\lambda_{p} K(n, 1)^{2}\right) .
$$

Since it is valid for any $\varepsilon>0$, we obtain $\liminf _{p \rightarrow 1} \lambda_{p} \geq K(n, 1)^{-2}$. By (2.5), we get that (2.8) is proved. Then (2.9) is an obvious consequence of $(2.3),(2.4),(2.7)$ and (2.8).

Step 2. We let $\Omega_{p}=\mu_{p}^{-1} \exp _{x_{p}}^{-1}\left(B_{g}\left(0, r_{p}\right)\right) \subset \mathbf{R}^{n}$ and we set

$$
g_{p}(x)=\exp _{x_{p}}^{\star} g\left(\mu_{p} x\right) \quad \text { for } x \in \Omega_{p}
$$

and

$$
v_{p}(x)=\mu_{p}^{\frac{n}{p}-1} u_{p}\left(\exp _{x_{p}}\left(\mu_{p} x\right)\right) \quad \text { for } x \in \Omega_{p}, v_{p}(x)=0 \quad \text { for } x \in \mathbf{R}^{n} \backslash \Omega_{p} .
$$

Clearly we have

$$
C_{p} \Delta_{p, g_{p}} v_{p}+\alpha \mu_{p}^{2}\left\|v_{p}\right\|_{p}^{2-p} v_{p}^{p-1}=\lambda_{p} v_{p}^{p^{\star}-1} \quad \text { in } \Omega_{p}
$$

with $v_{p}=0$ on $\partial \Omega_{p}$ and

$$
\int_{\Omega_{p}} v_{p}^{p^{\star}} d v_{g_{p}}=1
$$

We also let

$$
\tilde{v}_{p}(x)=v_{p}(x)^{\frac{p(n-1)}{n-p}} .
$$

By the Cartan expansion of a metric in the exponential chart, there exists $C>1$ such that

$$
\begin{aligned}
d v_{g_{p}} & \geq\left(1-\frac{1}{C} \mu_{p}^{2}\right) d v_{\xi}, \\
\left|\nabla \tilde{v}_{p}\right|_{g_{p}} d v_{g_{p}} & \leq\left(1+C \mu_{p}^{2}\right)\left|\nabla \tilde{v}_{p}\right|_{\xi} d v_{\xi}
\end{aligned}
$$

where $\xi$ is the Euclidean metric. This easily leads with (2.9), (2.11) and Hölder's inequalities to

$$
\lim _{p \rightarrow 1} \frac{\int_{\mathbf{R}^{n}}\left|\nabla \tilde{v}_{p}\right|_{\xi}^{p} d v_{\xi}}{\left(\int_{\mathbf{R}^{n}} v_{p}^{p^{\star}} d v_{\xi}\right)^{\frac{n-1}{n}}}=K(n, 1)^{-1} .
$$


Remember here that $r_{p} \rightarrow 0$ as $p \rightarrow 1$. Since $\left(\tilde{v}_{p}\right)$ is bounded in $H_{1}^{1}\left(\mathbf{R}^{n}\right)$, there exists $v_{0} \in B V\left(\mathbf{R}^{n}\right)$ such that

$$
\lim _{p \rightarrow 1} \tilde{v}_{p}=v_{0} \quad \text { weakly in } B V\left(\mathbf{R}^{n}\right) .
$$

If we apply the concentration-compactness principle of P.L. Lions (11], [12, see also [14 for an exposition in book form) to $\left|v_{p}\right|^{\star} d v_{\xi}$, four situations may occur: compactness, concentration, dichotomy or vanishing. Dichotomy is classically forbidden by (2.13). Concentration cannot happen since $\sup _{\Omega_{p}} v_{p}=v_{p}(0)=1$. As for vanishing, since $v_{p}$ is bounded in $L^{\infty}$, by applying Moser's iterative scheme to (2.10), one gets the existence of some $C>0$ such that for any $p>1$,

$$
1=\sup _{\Omega_{p} \cap B_{g_{p}}(0,1 / 2)} v_{p} \leq C\left(\int_{\Omega_{p} \cap B_{g_{p}}(0,1)} v_{p}^{p^{\star}} d v_{\xi}\right)^{\frac{1}{p^{\star}}} .
$$

Thus vanishing cannot happen. Compactness together with (2.13) just gives

$$
\lim _{p \rightarrow 1} \tilde{v}_{p}=v_{0} \text { strongly in } B V\left(\mathbf{R}^{n}\right) .
$$

Then $v_{0}$ is a minimizer for the $H_{1}^{1}$ Euclidean Sobolev inequality which verifies $\int_{\mathbf{R}^{n}} v_{0}^{\frac{n}{n-1}} d v_{\xi}=1$. Thus there exists $y_{0} \in \mathbf{R}^{n}, \lambda_{0}>0$ and $R_{0}>0$ such that

$$
v_{0}=\lambda_{0} \mathbf{1}_{B\left(y_{0}, R_{0}\right)}
$$

where $\mathbf{1}_{B\left(y_{0}, R_{0}\right)}$ denotes the characteristic function of the Euclidean ball $B\left(y_{0}, R_{0}\right)$. Moreover, since $v_{p} \leq 1$ in $\Omega_{p}$, we obtain with (2.14) that $v_{p} \rightarrow v_{0}$ in any $L^{q}\left(\mathbf{R}^{n}\right)$, $q \geq \frac{n}{n-1}$. One can deduce from this that $\lambda_{0}=1$. At last, we have:

$$
\operatorname{Vol}_{\xi}\left(B\left(y_{0}, R_{0}\right)\right)=\frac{\omega_{n-1}}{n} R_{0}^{n}=1 .
$$

Up to changing $x_{p}$ into $\exp _{x_{p}}\left(\mu_{p} y_{0}\right)$ in the definition of $v_{p}, \Omega_{p}$ and $g_{p}$, we may assume that $y_{0}=0$. We have thus obtained that

$$
\lim _{p \rightarrow 1} \tilde{v}_{p}=\mathbf{1}_{B\left(0, R_{0}\right)} \text { strongly in } B V\left(\mathbf{R}^{n}\right) .
$$

This means in particular that

$$
\lim _{p \rightarrow 1} \tilde{v}_{p}=\mathbf{1}_{B\left(0, R_{0}\right)} \quad \text { strongly in } L^{\frac{n}{n-1}}\left(\mathbf{R}^{n}\right)
$$

and that for any $\varphi \in C_{c}^{\infty}\left(\mathbf{R}^{n}\right)$,

$$
\lim _{p \rightarrow 1} \int_{\mathbf{R}^{n}}\left|\nabla \tilde{v}_{p}\right|_{\xi} \varphi d v_{\xi}=\int_{\partial B\left(0, R_{0}\right)} \varphi d \sigma_{\xi}
$$

If we set

$$
V_{p}(x)=\left(1+\left(\frac{|x|}{R_{0}}\right)^{\frac{p}{p-1}}\right)^{1-n}, x \in \mathbf{R}^{n},
$$

a simple application of the concentration-compactness principle, using what we just proved, gives

$$
\lim _{p \rightarrow 1} \int_{\mathbf{R}^{n}}\left|\nabla\left(\tilde{v}_{p}-V_{p}\right)\right|_{\xi} d v_{\xi}=0 .
$$


Applying Moser's iterative scheme to (2.10) with the help of (2.17), we also get that for any $R>R_{0}$,

$$
\lim _{p \rightarrow 1} \sup _{\Omega_{p} \backslash B(0, R)} v_{p}=0 .
$$

Step 3. The aim is to transform the $L^{\frac{n}{n-1}}$-estimate (2.17) into a pointwise estimate. We follow here [6] (see also [5]). We let

$$
w_{p}(z)=|z|^{\frac{n}{p}-1} v_{p}(z)
$$

and we let $z_{p} \in \Omega_{p}$ be a point where $w_{p}$ achieves its maximum. Let us assume by contradiction that

$$
\lim _{p \rightarrow 1} w_{p}\left(z_{p}\right)=+\infty
$$

We set

$$
\nu_{p}^{1-\frac{n}{p}}=v_{p}\left(z_{p}\right)
$$

so that

$$
\lim _{p \rightarrow 1} \frac{\left|z_{p}\right|}{\nu_{p}}=+\infty .
$$

Independently, since $v_{p} \leq 1$ in $\Omega_{p}$,

$$
\lim _{p \rightarrow 1}\left|z_{p}\right|=+\infty .
$$

Thanks to (2.22) and (2.23), one proves then that $\left(\nu_{p}^{\frac{n}{p}-1} v_{p}\left(\exp _{z_{p}}\left(\nu_{p} x\right)\right)\right)$ is bounded in $L^{\infty}(B(0,1))$. This allows us to apply Moser's iterative scheme to the equation verified by $\left(\nu_{p}^{\frac{n}{p}-1} v_{p}\left(\exp _{z_{p}}\left(\nu_{p} x\right)\right)\right)$ and to get the existence of some $C>0$ such that

$$
\liminf _{p \rightarrow 1} \int_{B_{g_{p}}\left(z_{p}, \nu_{p}\right) \cap \Omega_{p}} v_{p}^{p^{\star}} d v_{g}>0
$$

The contradiction then easily follows from $(2.17),(2.22)$ and (2.23). Thus we have the existence of some $C>0$ such that for any $p>1$, any $z \in \Omega_{p}$,

$$
|z|^{\frac{n}{p}-1} v_{p}(z) \leq C .
$$

In the same way, using (2.24), one proves thanks to (2.21) that for any $R>R_{0}$,

$$
\lim _{p \rightarrow 1} \sup _{\Omega_{p} \backslash B_{g_{p}}(0, R)}|z|^{\frac{n}{p}-1} v_{p}(z)=0 .
$$

We refer the reader to [6] for details on such claims.

Step 4 . We let $L_{p}$ be the following operator:

$$
L_{p} u=C_{p} \Delta_{p, g_{p}} u+\alpha \mu_{p}^{2}\left\|v_{p}\right\|_{p}^{2-p} u^{p-1}-\lambda_{p} v_{p}^{p^{*}-p} u^{p-1} .
$$

We fix $0<\nu<n-1$ and we set

$$
G_{p}(x)=\theta_{p}|x|^{-\frac{n-p-\nu}{p-1}}
$$


where $\theta_{p}$ is some positive constant to be fixed later. Easy computations lead to

$$
\begin{aligned}
|x|^{n-\nu} \frac{L_{p} G_{p}(x)}{G_{p}(x)^{p-1}} \geq & C_{p} \nu\left(\frac{n-p-\nu}{p-1}\right)^{p-1}-C \mu_{p}^{2}|x|^{2} \\
& +\alpha \mu_{p}^{2}\left\|v_{p}\right\|_{p}^{2-p}|x|^{p}-\lambda_{p}|x|^{p} v_{p}^{p^{\star}-p}
\end{aligned}
$$

in $\Omega_{p} \backslash\{0\}$. Here $C$ denotes some constant independent of $p$. Thanks to (2.7), (2.8), (2.9), (2.25) and the fact that $r_{p} \rightarrow 0$ as $p \rightarrow 1$, one gets that for any $R>R_{0}$,

$$
L_{p} G_{p}(x) \geq 0 \text { in } \Omega_{p} \backslash B_{g_{p}}(0, R)
$$

for $p$ small enough. On the other hand,

$$
L_{p} v_{p}=0 \text { in } \Omega_{p} .
$$

At last, it is not difficult to check with (2.21) that

$$
v_{p} \leq \theta_{p} G_{p} \quad \text { on } \partial B_{g_{p}}(0, R)
$$

if we take $\theta_{p}=R^{\frac{n-p-\nu}{p-1}}$. Now we may apply the maximum principle as stated for instance in [2] (lemma 3.4) to get, for $p$ small enough,

$$
v_{p}(y) \leq\left(\frac{R}{|y|}\right)^{\frac{n-p-\nu}{p-1}} \quad \text { in } \Omega_{p} \backslash B_{g_{p}}(0, R) .
$$

Since this inequality obviously holds on $B_{g_{p}}(0, R)$, we have finally obtained the following: for any $\nu>0$ and any $R>R_{0}$, there exists $C(R, \nu)>0$ such that for any $p>1$ and any $y \in \Omega_{p}$,

$$
\left(\frac{|y|}{R}\right)^{\frac{n-p-\nu}{p-1}} v_{p}(y) \leq C(R, \nu)
$$

Step 5. We conclude the proof of the Proposition. We apply the $H_{1}^{1}$ Euclidean Sobolev inequality to $\tilde{v}_{p}$ :

$$
\left(\int_{\Omega_{p}} \tilde{v}_{p}^{\frac{n}{n-1}} d v_{\xi}\right)^{\frac{n-1}{n}} \leq K(n, 1) \int_{\Omega_{p}}\left|\nabla \tilde{v}_{p}\right|_{\xi} d v_{\xi}
$$

By the Cartan expansion of $g_{p}$ around 0, we have

$$
d v_{\xi}=\left(1+\frac{\mu_{p}^{2}}{6} \operatorname{Ric}_{g}\left(y_{p}\right)_{i j} x^{i} x^{j}+o\left(\mu_{p}^{2}|x|^{2}\right)\right) d v_{g_{p}}
$$

where $R i c_{g}$ denotes the Ricci curvature of $g$ in the $\exp _{y_{p}}$-map. Thus, by (2.11),

$$
\int_{\Omega_{p}} \tilde{v}_{p}^{\frac{n}{n-1}} d v_{\xi}=1+\frac{\mu_{p}^{2}}{6} \operatorname{Ric}_{g}\left(y_{p}\right)_{i j} \int_{\Omega_{p}} x^{i} x^{j} v_{p}^{p^{\star}} d v_{g_{p}}+o\left(\mu_{p}^{2} \int_{\Omega_{p}}|x|^{2} v_{p}^{p^{\star}} d v_{g_{p}}\right) .
$$

Using (2.17) and (2.26), one gets

$$
\int_{\Omega_{p}} \tilde{v}_{p}^{\frac{n}{n-1}} d v_{\xi}=1+\frac{S_{g}(0)}{6 n(n+2)} \omega_{n-1} R_{0}^{n+2} \mu_{p}^{2}+o\left(\mu_{p}^{2}\right) .
$$

By the Cartan expansion of $g_{p}$ around 0 , since $r_{p} \rightarrow 0$ as $p \rightarrow 1$, we also have

$$
\left|\nabla \tilde{v}_{p}\right|_{\xi}^{p}=\left|\nabla \tilde{v}_{p}\right|_{g_{p}}^{p}\left[1-\frac{\mu_{p}^{2}}{6}\left|\nabla \tilde{v}_{p}\right|_{g_{p}}^{-2} R m_{g}\left(y_{p}\right)\left(\nabla \tilde{v}_{p}, x, x, \nabla \tilde{v}_{p}\right)+o\left(\mu_{p}^{2}|x|^{2}\right)\right]
$$


where $R m_{g}$ denotes the Riemann curvature of $g$ in the $\exp _{y_{p}}$-map. Then, using (2.28), we get

$$
\begin{aligned}
\int_{\Omega_{p}}\left|\nabla \tilde{v}_{p}\right|_{\xi} d v_{\xi}= & \int_{\Omega_{p}}\left|\nabla \tilde{v}_{p}\right|_{g_{p}} d v_{g_{p}}+\frac{\mu_{p}^{2}}{6} R i c_{g}\left(y_{p}\right)_{i j} \int_{\Omega_{p}} x^{i} x^{j}\left|\nabla \tilde{v}_{p}\right|_{\xi} d v_{\xi} \\
& -\frac{\mu_{p}^{2}}{6} \int_{\Omega_{p}}\left|\nabla \tilde{v}_{p}\right|_{g_{p}}^{-1} R m_{g}\left(y_{p}\right)\left(\nabla \tilde{v}_{p}, x, x, \nabla \tilde{v}_{p}\right) d v_{g_{p}} \\
& +o\left(\mu_{p}^{2} \int_{\Omega_{p}}|x|^{2}\left|\nabla \tilde{v}_{p}\right|_{g_{p}} d v_{g_{p}}\right) .
\end{aligned}
$$

Let us now look at the different terms of (2.30). First, by equation (2.10) and relation $(2.5)$, we have

$$
\begin{aligned}
\int_{\Omega_{p}}\left|\nabla \tilde{v}_{p}\right|_{g_{p}} d v_{g_{p}} & =\frac{p(n-1)}{n-p} \int_{\Omega_{p}} v_{p}^{\frac{n(p-1)}{n-p}}\left|\nabla v_{p}\right|_{g_{p}} d v_{g_{p}} \\
& \leq \frac{p(n-1)}{n-p}\left(\int_{\Omega_{p}}\left|\nabla v_{p}\right|_{g_{p}}^{p} d v_{g_{p}}\right)^{\frac{1}{p}} \\
& \leq K(n, 1)^{-1}\left(1-\alpha \mu_{p}^{2} \lambda_{p}^{-1}\left\|v_{p}\right\|_{p}^{2}\right)^{\frac{1}{2}} .
\end{aligned}
$$

Since, by (2.17) and (2.26), $\left\|v_{p}\right\|_{p}=1+o(1)$, we get

$$
\int_{\Omega_{p}}\left|\nabla \tilde{v}_{p}\right|_{g_{p}} d v_{g_{p}} \leq K(n, 1)^{-1}-\frac{\alpha}{2} K(n, 1) \mu_{p}^{2}+o\left(\mu_{p}^{2}\right) .
$$

Independently, by Hölder's inequalities, we have

$$
\int_{\Omega_{p}}|x|^{2}\left|\nabla \tilde{v}_{p}\right|_{g_{p}} d v_{g_{p}} \leq \frac{p(n-1)}{n-p}\left(\int_{\Omega_{p}}|x|^{2 p}\left|\nabla v_{p}\right|_{g_{p}}^{p} d v_{g_{p}}\right)^{\frac{1}{p}} .
$$

By equation (2.10), one gets

$$
\begin{aligned}
\int_{\Omega_{p}}|x|^{2 p}\left|\nabla v_{p}\right|_{g_{p}}^{p} d v_{g_{p}} \leq & \int_{\Omega_{p}}\left|\nabla v_{p}\right|_{g_{p}}^{p-2}\left(\nabla\left(|x|^{2 p} v_{p}\right), \nabla v_{p}\right)_{g_{p}} d v_{g_{p}} \\
& +C \int_{\Omega_{p}}|x|^{2 p-1}\left|\nabla v_{p}\right|_{g_{p}}^{p-1} v_{p} d v_{g_{p}} \\
\leq & C+C\left(\int_{\Omega_{p}}|x|^{2 p}\left|\nabla v_{p}\right|_{g_{p}}^{p} d v_{g_{p}}\right)^{\frac{p-1}{p}}\left(\int_{\Omega_{p}}|x|^{p} v_{p}^{p} d v_{g_{p}}\right)^{\frac{1}{p}}
\end{aligned}
$$

where $C$ denotes some constant independent of $p$. Using (2.26) and Young's inequalities, one deduces that

$$
\int_{\Omega_{p}}|x|^{2 p}\left|\nabla v_{p}\right|_{g_{p}}^{p} d v_{g_{p}}=O(1)
$$

Now, for some $R>R_{0}$, we get by (2.18) that

$$
\int_{\Omega_{p}}\left|\nabla \tilde{v}_{p}\right|_{\xi} x^{i} x^{j} d v_{\xi}=O\left(\int_{\Omega_{p} \backslash B(0, R)}|x|^{2}\left|\nabla \tilde{v}_{p}\right|_{\xi} d v_{\xi}\right)+\int_{\partial B\left(0, R_{0}\right)} x^{i} x^{j} d \sigma_{\xi}+o(1) .
$$


Using equation (2.10) and relation (2.26), it is easy to check that

so that

$$
\lim _{p \rightarrow 1} \int_{\Omega_{p} \backslash B(0, R)}|x|^{2}\left|\nabla \tilde{v}_{p}\right|_{\xi} d v_{\xi}=0
$$

$$
\lim _{p \rightarrow 1} R i c_{g}\left(y_{p}\right)_{i j} \int_{\Omega_{p}}\left|\nabla \tilde{v}_{p}\right|_{\xi} x^{i} x^{j} d v_{\xi}=\frac{\omega_{n-1}}{n} R_{0}^{n+1} S_{g}(0) .
$$

At last, since $\nabla V_{p}, V_{p}$ as in (2.19), and $x$ are pointwise colinear vector fields, we have

$$
R m_{g}\left(y_{p}\right)\left(\nabla \tilde{v}_{p}, x, x, \nabla \tilde{v}_{p}\right) \leq C|x|^{2}\left|\nabla \tilde{v}_{p}\right|_{\xi}\left|\nabla\left(\tilde{v}_{p}-V_{p}\right)\right|_{\xi}
$$

so that, by (2.10), (2.20) and (2.26),

$$
\lim _{p \rightarrow 1} \int_{\Omega_{p}}\left|\nabla \tilde{v}_{p}\right|_{g_{p}}^{-1} R m_{g}\left(y_{p}\right)\left(\nabla \tilde{v}_{p}, x, x, \nabla \tilde{v}_{p}\right) d v_{g_{p}}=0 .
$$

Coming back to (2.27) with (2.29)-(2.34), we obtain, after easy computations using in particular (2.16),

$$
\left(\alpha-\frac{n}{n+2} S_{g}(0)\right) \mu_{p}^{2}+o\left(\mu_{p}^{2}\right) \leq 0 .
$$

This gives the desired contradiction by letting $p$ go to 0 . Remember here that $\alpha-\frac{n}{n+2} S_{g}(0)=\varepsilon_{0}>0$. This ends the proof of the Proposition, hence the proof of Theorem 1 .

\section{The compact case - Proof of Theorem 2}

In order to prove Theorem 2 , we let $(M, g)$ be a compact Riemannian manifold of dimension $n \geq 2$. We assume that $S_{g}<n(n-1) K_{0}$. If we apply Theorem 1 with some $x$ in $M$ and $K_{0}$, we get some $r_{x}>0$ such that the isoperimetric comparison (with the model space form of curvature $K_{0}$ ) holds for sets contained in the geodesic ball of center $x$ and radius $r_{x}$. It is clear that $r_{x}$ is continuous with respect to $x$. Thus, there exists $d>0$ such that for any subset $\Omega$ of $M$ of diameter less than or equal to $d$,

$$
|\partial \Omega|_{g}>|\partial B|_{g_{0}}
$$

where $B$ is a ball of volume $|\Omega|_{g}$ in the model space of constant curvature $K_{0}$.

For $0<V<|M|_{g}$, we let

$$
h(V)=\inf \left\{|\partial \Omega|_{g}, \Omega \subset M,|\Omega|_{g}=V\right\} .
$$

There exists some $\Omega_{V} \subset M$ such that

$$
\left|\partial \Omega_{V}\right|_{g}=h(V) .
$$

The boundary $\partial \Omega_{V}$ of $\Omega_{V}$ is a smooth hypersurface of constant mean curvature up to a compact set of Hausdorff dimension at most $n-8$ (see for instance [13]). Now, as a consequence of the work of Johnson and Morgan [10, we know that

$$
\operatorname{diam}\left(\Omega_{V}\right) \rightarrow 0
$$

as $V \rightarrow 0$. In fact, Johnson and Morgan proved that $\Omega_{V}$ is asymptotically, as $V \rightarrow 0$, a ball. In particular, for some $V_{0}$ small enough, any $\Omega_{V}$ for $V \leq V_{0}$ has a diameter less than or equal to $d$. We may then apply (3.1) to end the proof of Theorem 2. 


\section{ACKNOWLEDGEMENTS}

It is my pleasure to express my deep thanks to E. Hebey for his encouragement and helpful comments during the preparation of this work, and to G. Huisken for having pointed out to me the very nice Johnson and Morgan [10].

\section{REFERENCES}

1. T. Aubin, Problèmes isopérimétriques et espaces de Sobolev, J. Differential Geom. 11 (1976), 573-598. MR 56:6711

2. T. Aubin and Y.Y. Li, On the best Sobolev inequality, J. Math. Pures Appl. 78 (1999), 353387. MR 2000e:46041

3. A. Beauville, Variétés Kähleriennes dont la première classe de Chern est nulle, J. Differential Geom. 18 (1983), 755-782. MR 86c:32030

4. O. Druet, Optimal Sobolev inequalities of arbitrary order on compact Riemannian manifolds, J. Funct. Anal. 159 (1998), 217-242. MR 99m:53076

5. - The best constants problem in Sobolev inequalities, Math. Ann. 314 (1999), 327-346. MR 2000d:58033

6. Isoperimetric inequalities on compact manifolds, Geometriae Dedicata (to appear).

7. L.C. Evans and R.F. Gariepy, Measure theory and fine properties of functions, Studies in Advanced Mathematics, CRC Press, 1992. MR 93f:28001

8. E. Hebey, Nonlinear analysis on manifolds: Sobolev spaces and inequalities, CIMS Lecture Notes, vol. 5, Courant Institute of Mathematical Sciences, 1999. MR 2000e:58011

9. Sharp Sobolev-Poincaré inequalities on compact Riemannian manifolds, Preprint (2000).

10. D. Johnson and F. Morgan, Some sharp isoperimetric theorems for Riemannian manifolds, Indiana University Math. Journal 49, 3 (2000), 1017-1041.CMP 2001:06

11. P.L. Lions, The concentration-compactness principle in the calculus of variations. The locally compact case. Part I, Ann. Inst. H. Poincaré 1 (1984), 109-145. MR 87e:49035a

12. Part I, Rev. Mat. Iberoamericano 1.1 (1985), 145-201. MR 87c:49007

13. F. Morgan, Geometric Measure Theory : a Beginner's Guide, Academic Press, 1995. MR 96c:49001

14. M. Struwe, Variational Methods, Ergebnisse der Mathematik und ihrer Grenzgebiete, 34, Springer, 1996. MR 98f:49002

15. G. Talenti, Best constants in Sobolev inequality, Ann. Math. Pura Appl. 110 (1976), 353-372. MR 57:3846

16. W.P. Ziemer, Weakly differentiable functions, Graduate Text in Mathematics, 120, SpringerVerlag, 1989. MR 91e:46046

Département de Mathématiques, Université de Cergy-Pontoise, Site de Saint-Martin, 2 avenue Adolphe Chauvin, 95302 Cergy-Pontoise cedex, France

E-mail address: 0livier.Druet@math.u-cergy.fr 